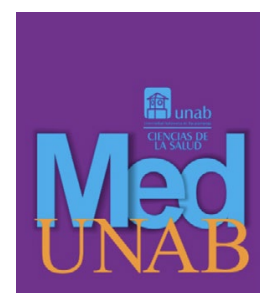

REVISTA DE LA FACULTAD

DE CIENCIAS DE LA SALUD

\title{
Utilidad de la evaluación USG Doppler de las arterias uterinas entre las semanas 11 y $13+6$ y su aplicación en las calculadoras de riesgo para predecir preeclampsia
}

Usefulness of the USG Doppler evaluation of the uterine arteries between weeks 11 and $13+6$ and its application in risk calculators to predict preeclampsia

Utilidade da avaliação USG Doppler das artérias uterinas entre as semanas 11 e $13+6$ e sua aplicação em calculadoras de risco para prever pré-eclâmpsia

María Camila Otero-Rosales, Est. ${ }^{1}$ (D), Cristhian David Olarte-Marín, Est. ${ }^{1}$ (D), Johan Danilo Padilla-Serpa, Est. ${ }^{1}$ (D), Paula Andrea Morales-Duarte, Est. ${ }^{1}$ (D), Eliana Maribel Quintero-Roa, MD., Esp., MSc. ${ }^{2}$ (D)

1. Estudiante de Medicina. Universidad Autónoma de Bucaramanga. Bucaramanga, Santander, Colombia.

2. Médico, Especialista en Ginecología y Obstetricia, Magister en Bioética, Docente. Universidad Autónoma de Bucaramanga. Bucaramanga, Santander, Colombia.

Correspondencia. María Camila Otero Rosales. Universidad Autónoma de Bucaramanga, Calle 157 \#154-215 Gaira, Cañaveral. Floridablanca, Santander, Colombia. Email: motero16@unab.edu.co

\section{INFORMACIÓN DEL ARTÍCULO:}

Artículo recibido: 21 de julio de 2020

Artículo aceptado: 12 de noviembre de 2021

DOI: https://doi.org/10.29375/01237047.3953

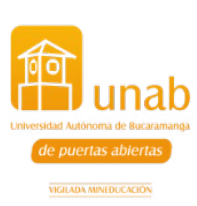

Cómo citar. Otero-Rosales MC, Olarte-Marín CD, Padilla-Serpa JD, MoralesDuarte PA, Quintero-Roa EM. Utilidad de la evaluación USG Doppler de las arterias uterinas entre las semanas 11 y $13+6$ y su aplicación en las calculadoras de riesgo para predecir preeclampsia. MedUNAB [Internet]. 2021;24(3):375383. doi: https://doi.org/10.29375/01237047.3953

\section{RESUMEN:}

Introducción. La preeclampsia es la primera causa de muerte materna directa en Colombia y la segunda a nivel mundial. El desarrollo de estrategias de predicción y prevención puede disminuir las complicaciones y secuelas ocasionadas por dicha enfermedad. El Doppler de arterias uterinas entre las semanas 11 y $13+6$ como 
prueba independiente o en combinación con factores maternos o pruebas bioquímicas permite tasas de detección de preeclampsia temprana $\geq 90 \%$ a partir de la implementación de distintos cribados. La validez de dicha prueba diagnóstica presenta una sensibilidad del $47.8 \%$ y especificidad del $92.1 \%$ para la detección de preeclampsia temprana; con una sensibilidad del $26.4 \%$ y especificidad del $93.4 \%$ para predecir preeclampsia en cualquier etapa. División de los temas tratados. En esta revisión de tema se aborda la utilidad de esta medición, se habla de la realización de la técnica en cuestión y, por último, se revisan las herramientas estandarizadas que están disponibles en la actualidad junto con su accesibilidad y precisión. Conclusiones. La evidencia empírica que respalda la validez de las herramientas disponibles hoy en día para el tamizaje de preeclampsia a través de la evaluación por ultrasonografía Doppler de las arterias uterinas es significativa. Al ser Colombia un país que presenta una prevalencia alta de preeclampsia, conocer la utilidad de esta medición favorece una vigilancia temprana y oportuna, lo que disminuye los posibles desenlaces desfavorables para las maternas.

Palabras claves:

Preeclampsia; Ultrasonografía; Ultrasonografía Prenatal; Arteria Uterina; Atención Prenatal; Diagnóstico Prenatal; Retardo del Crecimiento Fetal; Pruebas Prenatales no Invasivas.

\section{ABSTRACT}

Introduction. Preeclampsia is the primary cause of direct maternal death in Colombia and the second globally. The development of prediction and prevention strategies can reduce complications and consequences caused by this disease. The uterine arteries Doppler between weeks 11 and 13+6 as an independent test or in combination with maternal factors or biochemical tests allows for early detection rates for preeclampsia of $\geq 90 \%$ from the implementation of different sieving. The validity of this diagnostic test has a sensitivity of $47.8 \%$ and specificity of $92.1 \%$ for the early detection of preeclampsia; with a sensitivity of $26.4 \%$ and specificity of $93.4 \%$ to predict preeclampsia at any stage. Division of Covered Topics. This topic review covers the usefulness of this measurement. It discusses the performance of the technique in question and, lastly, the standardized tools currently available are reviewed together with the accessibility and accuracy. Conclusions. The empirical evidence that supports the validity of the tools available today for the screening of preeclampsia via Doppler ultrasound evaluation of the uterine arteries is significant. As Colombia is a country with a high prevalence of preeclampsia, knowing the usefulness of this measurement favors early and timely surveillance, which reduces possible unfavorable outcomes for mothers.

Keywords:

Pre-Eclampsia; Ultrasonography; Ultrasonography, Prenatal; Uterine Artery; Prenatal Care; Prenatal Diagnosis; Fetal Growth Retardation; Noninvasive Prenatal Testing.

\section{RESUMO}

Introdução. A pré-eclâmpsia é a principal causa de morte materna direta na Colômbia e a segunda no mundo. O desenvolvimento de estratégias de predição e prevenção pode reduzir as complicações e sequelas causadas pela doença. O Doppler da artéria uterina entre as semanas 11 e 13+6 como um teste independente ou em combinação com fatores maternos ou testes bioquímicos permite taxas de detecção de pré-eclâmpsia precoce $\geq 90 \%$ a partir da implementação de diferentes exames. A validade desse teste diagnóstico tem sensibilidade de $47,8 \%$ e especificidade de $92,1 \%$ para a detecção de pré-eclâmpsia precoce; com uma sensibilidade de $26,4 \%$ e especificidade de 93,4\% para prever pré-eclâmpsia em qualquer fase. Divisão dos tópicos abordados. Esta revisão de tópicos aborda a utilidade desta medição, discute a realização da técnica em questão e, por fim, são revisadas as ferramentas padronizadas que estão disponíveis atualmente, juntamente com sua acessibilidade e precisão. Conclusões. A evidência empírica que apoia a validade das ferramentas disponíveis atualmente para rastreamento de pré-eclâmpsia por meio da avaliação de ultrassom Doppler das artérias uterinas é significativa. Como a Colômbia é um país com alta prevalência de pré-eclâmpsia, conhecer a utilidade dessa medição favorece a vigilância precoce e oportuna, o que reduz possíveis resultados desfavoráveis para mulheres maternas.

Palavras-chave:

Pré-Eclâmpsia; Ultrassonografia; Ultrassonografia Pré-Natal; Artéria Uterina; Cuidado Pré-Natal; Diagnóstico Pré-Natal; Retardo do Crescimento Fetal; Teste Pré-Natal não Invasivo. 


\section{Introducción}

Los trastornos hipertensivos afectan hasta al 10\% de las mujeres embarazadas, prevalencia que puede ser mayor en países en vía de desarrollo. La incidencia global de la preeclampsia (PE) es aproximadamente del 3\% (1). Para el año 2019, según las estadísticas del Sistema Nacional de Vigilancia en Salud Pública (SIVIGILA), cuyo fin es proveer de forma sistemática información sobre la dinámica de los eventos que afectan la salud de la población en Colombia, es la primera causa de muerte materna directa y la segunda a nivel mundial (2). La preeclampsia es una enfermedad multisistémica, que se manifiesta después de la semana 20 y se caracteriza por hipertensión $(>140 / 90$ $\mathrm{mmHg}$ ) y proteinuria $(>300 \mathrm{mg}$ en orina $24 \mathrm{~h} \mathrm{o} \geq++$ tiras reactivas) (1), la cual puede ser la causante de múltiples desenlaces como retardo del crecimiento intrauterino (RCIU), parto prematuro, desprendimiento de placenta, entre otros, que generan gran mortalidad y morbilidad materna y neonatal (3). El fundamento del cribado de la PE en las semanas 11 y $13+6$ es que desde estas semanas ya se pueden evidenciar los cambios generados por el proceso fisiopatológico de la enfermedad en un estadio temprano, lo que implica la capacidad de predecir las complicaciones posteriores del embarazo, y permite el inicio temprano de una terapia profiláctica y mejorar la vigilancia clínica en la población de riesgo verdaderamente alto (3). Dado que la atención oportuna y efectiva puede disminuir las complicaciones y secuelas de la preeclampsia en el binomio madre-hijo, el desarrollo de estrategias de predicción y prevención ha sido una de las principales finalidades de la atención prenatal y de la investigación (4). El objetivo de este artículo es revisar la literatura científica acerca del uso de la ultrasonografía (USG) Doppler de las arterias uterinas entre las semanas 11 y $13+6$ y su utilidad en asociación con las calculadoras de riesgo para la predicción de PE con el fin de implementarlas en la atención primaria.

\section{División de los temas tratados}

Todos los artículos usados para la realización de este texto se han obtenido de Embase, SciELO, PubMed y Cochrane Library, publicados entre los años 2000 a 2020; se tuvieron en cuenta estudios en inglés y español. Se han incluido estudios de pruebas diagnósticas, estudios observacionales y metaanálisis de los mismos, se descartaron estudios en los que no se tuvieran en cuenta gestantes entre las 11 y $13+6$ semanas. Inicialmente se encontraron 50 artículos, posterior a una revisión se decide hacer uso de 41 artículos que son los más pertinentes con respecto al tema. El artículo aborda la historia del Doppler de arterias uterinas, enfocándose en la manera como se llevaron a cabo los primeros hallazgos y cómo estos se empezaron a relacionar con el desenlace de PE; en qué consiste la técnica de revisión y cómo se realiza en términos generales, y los algoritmos que se usan actualmente como calculadoras de predicción de riesgo.

\section{Historia}

Una de las propuestas de predicción establecidas es el uso de la velocimetría de las arterias uterinas a partir del Doppler como prueba independiente o en combinación con factores maternos o pruebas bioquímicas. El Doppler de arterias uterinas fue descrito por primera vez en 1983, como un medio para la predicción de PE (5). En este estudio se compararon embarazos con ondas de Doppler normales y anormales, en estos últimos se encontró mayor asociación con las patologías mencionadas. El parámetro de medición utilizado fue la evaluación del índice de resistencia y el hallazgo consistió en un aumento de su valor cuando se realizaba en el tercer trimestre de gestación. Este hallazgo despertó gran interés en la posible utilidad diagnóstica de esta prueba, lo que impulsó la realización de nuevas investigaciones (6). Aproximadamente en el año 2000, Farré et al. (7) desarrollaron un nuevo estudio con un enfoque específico para esta prueba, con su aplicación en la evaluación de las arterias uterinas entre las semanas 19-25 de gestación. Se evaluaron 313 gestantes y se encontró que los principales factores predictores para PE eran el índice de resistencia (IR) elevado y la presencia del notch (incisura protodiastólica que se aprecia en las ondas sonoras de la evaluación Doppler) proto-diastólico. En este mismo periodo de tiempo, Papageorgiou et al. (8) proponen la utilización de este examen en el segundo trimestre de gestación, antes de las 23 semanas de embarazo. Se les realizó Doppler de arterias uterinas a 8,335 mujeres y se encontró que, aunque la sensibilidad de la presencia de muescas bilaterales fue similar a la del índice de pulsatilidad aumentado, y la tasa de detección positiva $(9.3 \%)$ fue mayor que el índice de pulsatilidad aumentado (5.1\%), estos indicadores eran hallazgos muy subjetivos, lo cual generaba una variabilidad interobservador elevada. Por esta causa, se estableció el índice de pulsatilidad (IP) promedio de las dos arterias uterinas con valor mayor al percentil 95 como el indicador con mayor relevancia para predecir la posibilidad de desarrollar PE temprana y RCIU. Martin et al. (9) midieron los promedios de los 
IP de las ondas de velocidad de flujo de ambas arterias uterinas entre las semanas 11 a 14 y encontraron que un valor de IP $>$ P95 tuvo una sensibilidad de detección del $60 \%$ para $\mathrm{PE}$.

El desempeño de la prueba como predictor del desarrollo de preeclampsia ha sido estudiado en diversas investigaciones. En el metaanálisis realizado por Velauthar et al. (10) se encontró que el examen Doppler de primer trimestre de las arterias uterinas en gestantes con bajo riesgo tiene una sensibilidad del 47.8\% (IC 95\%: 39.0-56.8) y una especificidad del 92.1\% (IC 95\%: 88.6-94.6) en los casos de PE temprana (tasa de falsos positivos [TFP] del 7.9\%), y una sensibilidad de 26.4\% (IC 95\%: 22.5-30.8) y especificidad del 93.4\% (IC 95\% 90.4-95.5) para predecir PE en cualquier etapa (tasa TFP de 6.6\%) de la gestación, al utilizarse como punto de corte el percentil 90 de la medición del índice de pulsatilidad de las arterias uterinas (IP-AtU) (Tabla 1) (10). La prevalencia de preeclampsia de inicio temprano varía del 0.4 al $1 \%$ en la población general. En mujeres de bajo riesgo con Doppler anormal de la arteria uterina en el primer trimestre, el riesgo de preeclampsia de inicio temprano varía entre 2.4 y $5.8 \%$ para la prevalencia variable de la enfermedad, similar al de mujeres con un factor de alto riesgo, que varía de 1.5 a $3.9 \%$ (11).

Tabla 1. Estimaciones de precisión de la medición del Doppler de arterias uterinas en primer trimestre en gestantes con bajo riesgo de preeclampsia

\begin{tabular}{|c|c|c|c|c|}
\hline Resultados & $\begin{array}{c}\text { Doppler } \\
\text { Test }\end{array}$ & $\begin{array}{c}\text { Sensibilidad } \\
\text { (\% [IC } 95 \% \\
\text { IC] })\end{array}$ & $\begin{array}{c}\text { Especificidad } \\
\text { (\% [IC } 95 \% \\
\text { IC]) }\end{array}$ & $\begin{array}{c}\text { Tasa de } \\
\text { falsos } \\
\text { positivos } \\
\text { (TFP) }\end{array}$ \\
\hline $\begin{array}{c}\text { PE } \\
\text { temprana }\end{array}$ & IP-AtU & $\begin{array}{c}47.8 \% \\
\text { (IC 95\%: } \\
39.0-56.8)\end{array}$ & $\begin{array}{c}92.1 \% \\
\text { (IC 95\%: } \\
88.6-94.6)\end{array}$ & $7.9 \%$ \\
\hline $\begin{array}{c}\text { PE } \\
\text { cualquier } \\
\text { etapa de } \\
\text { gestación }\end{array}$ & IP-AtU & $\begin{array}{c}26.4 \% \\
\text { (IC 95\%: } \\
22.5-30.8)\end{array}$ & $\begin{array}{c}93.4 \\
\text { (IC 95\%: } \\
90.4-95.5)\end{array}$ & $6.6 \%$ \\
\hline
\end{tabular}

Se ilustran las estimaciones de precisión de la medición del IP-AtU por Doppler en gestantes con bajo riesgo de preeclampsia. TFP: Tasa de falsos positivos; IP-AtU: índice de pulsatilidad de las arterias uterinas; PE: preeclampsia.

Fuente: adaptado de: Velauthar L, Plana MN, Kalidindi M, Zamora J, Thilaganathan B, Illanes SE, et al. First-trimester uterine artery Doppler and adverse pregnancy outcome: a meta-analysis involving 55974 women. Ultrasound Obstet Gynecol [Internet]. 2014; 43: 500-507. doi: https://doi.org/10.1002/uog.13275 (10)
El uso de la medición de la velocimetría de las arterias uterinas a partir del Doppler se correlaciona con la patogénesis de la preeclampsia, pues evidencia el aumento de la impedancia de las arterias uterinas que tiene su origen en la insuficiencia placentaria ocasionada por la invasión trofoblástica deficiente de las arterias espirales maternas. Esta explicación etiológica está fuertemente asociada con la mayoría de los casos de PE temprana y grave $(12,13)$. Si se confirma la alteración de la impedancia en las arterias uterinas en el primer trimestre, se podrá establecer un manejo preventivo óptimo (14). Actualmente, el Doppler de arterias uterinas es el examen no invasivo más utilizado como método predictor para PE cuando se evalúa entre las semanas 11-14 de gestación.

En aquellos embarazos que desarrollan PE, la medición del IP-AtU y de la presión arterial media (PAM) a las 11-14 semanas de gestación aumentan, y los valores de proteína plasmática - A asociada al embarazo (PAPP-A) y el factor de crecimiento placentario sérico (FCPS) en suero disminuyen (15).

\section{Técnica de la medición}

Considerando la alta utilidad de este método, es pertinente describir la técnica de su realización. Esta se puede efectuar tanto por vía transabdominal (TA) como transvaginal (TV). Ambas han demostrado tener reproducibilidad a la hora de medir el IP-AtU, sin embargo, la que posee mayor sensibilidad $y$ especificidad en la edad gestacional planteada es aquella que se realiza por vía TV $(16,17)$.

La técnica indica que se debe obtener un corte sagital del útero y con la ayuda del Doppler de color se identifica el trayecto de la arteria uterina y se toman las mediciones: índice de resistencia (IR) e índice de pulsatilidad (IP) justo antes de su ramificación en arterias arcuatas $(18,19)$. Este proceso se debe hacer de manera estandarizada para garantizar la precisión de los resultados, por ende, debe ser realizado por personal capacitado (20).

La aplicación de esta técnica implica que, para utilizar esta información como predictor de la posibilidad de desarrollar PE, se deben conocer los valores normales de cada uno de estos parámetros, con el fin de identificar las anomalías que se presenten. Tayyar et al. (21), en un estudio realizado a 940 participantes, midieron y promediaron el IP de las arterias uterinas derecha e izquierda y obtuvieron valores de índice de pulsatilidad medio (IPm) que disminuían conforme la edad 
gestacional avanzaba. Rivas et al. (22) establecieron, en 559 gestantes, los valores de referencia del IPAtU promedio entre las semanas 11-40 de gestación: encontraron valores entre 0.70 (P5) a 1.42 (P50) en las semanas 11-14.

En el estudio realizado por Plasencia et al. (16) en Canarias con 351 pacientes, se encontró que el IP-AtU medido por ultrasonido TA fue de 1.83 (IC 95\%: 1.78 1.89), mientras que el medido por ultrasonido TV fue significativamente mayor, con un IPm de 1.98 (IC 95\%: 1.93-2.08) ( $<$ 0.05). Los datos mostraron que el IPm (medido tanto por ultrasonido TA como por ultrasonido TV) disminuyó a medida que aumentó la longitud craneocaudal (LCC). El ultrasonido TA mostró que el IPm disminuyó progresivamente a medida que aumentó el tamaño del feto, de 1.96 (IC 95\%: 1.80-2.12) para fetos más pequeños con un LCC $<60 \mathrm{~mm}$, a 1.71 (IC 95\%: 1.56-1.87) para los más grandes, con un LCC $\geq 70$ $\mathrm{mm}(\mathrm{p}<0.05)$. El ultrasonido TV mostró una tendencia similar, con valores de IPm de 2.09 (IC 95\%: 1.93-2.26) para fetos más pequeños y 1.78 (IC 95\%: 1.64-1.92) para los más grandes $(\mathrm{p}<0.05)$. Adicionalmente, Ferreira et al. (23), en un estudio observacional, corroboraron que el IP-AtU fue significativamente mayor cuando se midió por ultrasonido TV que por ultrasonido TA $(1.60 \pm 0.49$ vs. $1.52 \pm 0.63 ; \mathrm{P}=0.03)$.

De acuerdo con la evidencia mencionada, la técnica transvaginal se caracteriza por lecturas significativamente más altas en comparación con la técnica transabdominal; la razón de esto puede ser que la ecografia transvaginal permite una proximidad más cercana del transductor al vaso, incluso se puede ubicar el Doppler en la arteria uterina principal antes de su ramificación $(23,24)$. Además, el examen uterino transabdominal en las primeras etapas se dificulta debido a que el útero no es lo suficientemente grande y es necesaria la técnica de vejiga completa lo que puede causar una alteración en el flujo sanguíneo en las arterias pequeñas (25).

\section{Calculadoras de riesgo de preeclampsia entre las semanas 11 a $13+6$}

Se han creado diversos algoritmos de evaluación del riesgo de PE en el primer trimestre debido a la utilidad del Doppler de arterias uterinas, los cuales son usados por software que, al insertar ciertas características de determinada paciente, arrojan un cálculo sobre el riesgo de preeclampsia individual. Dada la variedad de estos, el profesional de la salud debe conocer sus características para saber cuál usar en el cribado, en qué población aplicarlos y qué tan divergentes son sus resultados (26).

El algoritmo propuesto por O'Gorman et al. y adoptado por la Fetal Medicine Foundation (FMF) (27), construido a partir de una cohorte de 35,948 embarazadas en Inglaterra, abarca factores de riesgo maternos junto con la medición de la PAM, el Doppler que mide la IP-AtU y ciertos biomarcadores como el FCPS y el PAPP-A, por lo cual es denominado como el método de tamizaje triple test. En el estudio de su validación se demostró que el triple test tiene mayor tasa de detección que si se toman las mismas variables por separado, lo que muestra una tasa de detección (TD) de 75\% (IC 95\%: $70-80 \%$ ) para PE pretérmino, y de $47 \%$ (IC $95 \%$ : 44$51 \%$ ) para PE a término, ambos datos a una TFP de $10 \%$. Este algoritmo ha sido validado en diferentes muestras. Un estudio de los mismos autores (28), con 8,775 pacientes embarazadas de 5 países europeos, aplicó el mismo algoritmo para evaluar su precisión y obtuvo una TD de 100\% (IC 95\%: 80-100\%), $75 \%$ (IC 95\%: 62-85\%) y 43\% (IC 95\%: $35-50 \%$ ) para PE con trabajo de parto antes de las 32 y de las 37 semanas, y después de las 37 semanas, respectivamente, lo que demuestra la concordancia con los resultados mostrados en el estudio anterior.

Con base en el consenso actual sobre el tamizaje para $\mathrm{PE}$, el cual consiste únicamente en tener en cuenta los factores de riesgo maternos, O'Gorman et al. (29) compararon la sensibilidad de esta calculadora con los protocolos de tamizaje propuestos por el American College of Obstetricians and Gynecologists (ACOG) (30) y por el National Institute for Health and Care Excellence (NICE) (31) (Tabla 2) y encontraron que el tamizaje con el algoritmo de la FMF detectó el 100, 75 y $43 \%$ a una TFP de $10.0 \%$, para $\mathrm{PE}<32,<37$ y $\geq 37$ semanas, respectivamente. El tamizaje basado en las indicaciones de las guías NICE detectó 41,39 y $34 \%$ a una TFP de $10.2 \%$ para $\mathrm{PE}<32,<37 \mathrm{y} \geq 37$ semanas, respectivamente. Por último, el tamizaje basado en las recomendaciones del ACOG detectó 94, 90 y $89 \%$ a una TFP de $64.2 \%$, para $\mathrm{PE}<32,<37 \mathrm{y} \geq 37$ semanas, respectivamente. Se concluye que el algoritmo de la FMF es más eficaz para detectar PE que el tamizaje sustentado únicamente en las recomendaciones del ACOG y el NICE.

Otro algoritmo propuesto por Akolekar et al. (32), a partir de un estudio de 2013 con 58,884 pacientes en Reino Unido (de manera similar al algoritmo de FMF), utiliza el teorema de Bayes para combinar la información de las características maternas junto con la PAM, la medición del IP-AtU, la PAPP-A y 
la FCPS para generar una probabilidad y calcular la TD. Según los parámetros evaluados, se evidencia una TD de $93.4,61.1$ y $37.8 \%$ para PE con trabajo de parto $<34,<37$ y $<42$ semanas, respectivamente, al combinar todas las variables del tamizaje a una TFP del 5\%. Posteriormente, este algoritmo fue probado en la cohorte ASPRE (33), la cual contaba con una población de 25,797 pacientes de Reino Unido, España, Italia, Bélgica, Grecia e Israel, con el fin de clasificar a las pacientes en grupos según el riesgo de desarrollar PE y someterlas a un ensayo clínico con aspirina a dosis bajas. Los resultados, después de hacer un ajuste estadístico con base en la prescripción de aspirina de este estudio, mostraron una TD de 76.7\% para PE pretérmino y de $43.1 \%$ para PE a término, con una TFP de $9.5 \%$, con lo cual se hace factible la incorporación de este algoritmo en la práctica clínica.

Tabla 2. TD (\%) Preeclampsia con trabajo de parto según la semana de inicio

TD (\%) Preeclampsia con trabajo de parto según la
semana de inicio
\begin{tabular}{lccc} 
Método de tamizaje & $<\mathbf{3 2}$ & $<\mathbf{3 7}$ & $>\mathbf{0}=\mathbf{3 7}$ \\
semanas & semanas & semanas \\
\hline Algoritmo FMF & & & \\
\hline$(\mathrm{TFP}=10 \%)$ & & & \\
\hline
\end{tabular}

$\begin{array}{llll}\text { Factores maternos } & 53 & 41 & 37\end{array}$

Factores maternos más:

$\begin{array}{llll}\text { PAM } & 71 & 47 & 37 \\ \text { IP-AtU } & 82 & 61 & 39 \\ \text { PAM, IP-AtU } & 94 & 71 & 41 \\ \text { NICE }(\text { TFP }=10.2 \%) & 41 & 39 & 34 \\ \underline{\text { ACOG }(\text { TFP }=64.2 \%)} & 94 & 90 & 89\end{array}$

Se ilustran las tasas de detección(TD) según la semana en la que haya iniciado el cuadro de preeclampsia en el algoritmo de la Fetal Medicine Foundation. FMF: Fetal Medicine Foundation; TFP: Tasa de falsos positivos; PAM: presión arterial media; IP-AtU: índice de pulsatilidad de las arterias uterinas; NICE: National Institute for Health and Care Excellence; ACOG: American College of Obstetricians and Gynecologists.

Fuente: adaptado de: O'Gorman N, Wright D, Poon L, Rolnik D, Syngelaki A, de Alvarado M, et al. Multicenter screening for pre-eclampsia by maternal factors and biomarkers at 11-13 weeks' gestation: comparison with NICE guidelines and ACOG recommendations. Ultrasound Obstet Gynecol [Internet]. 2017;49(6):756-760. doi: https://doi.org/10.1002/ uog.17455(29)
De acuerdo con la similitud de las tasas de detección (TD) encontradas en diversas investigaciones, fue necesario la realización de un enfoque comparativo; ante esto, un estudio realizado por Skråstad et al. (34) evaluó la sensibilidad de dos algoritmos (el FMF y el Predictor) en población escandinava. Se encontró que los resultados obtenidos en su muestra, teniendo en cuenta que es una población en la que la tasa de PE es baja, tuvo resultados similares entre ambos algoritmos y concordantes con estudios previos en diferentes poblaciones. Otro estudio de 2018, realizado por Lakovschek et al. (35), tuvo como objetivo evaluar tres distintos algoritmos para el tamizaje PE en el primer trimestre de embarazo. Comparó el algoritmo de la FMF, el programa Predictor de PerkinElmer (PERK) y el algoritmo de ViewPoint (VP) para evaluar la concordancia de los resultados de los tres test a través de la Kappa de Cohen. Se evidenció una concordancia entre moderada y sustancial (PERK/VP: $\mathrm{K}=0.56$; PERK/FMF: $\mathrm{K}=0.50$; VP/FMF: $\mathrm{K}=0.72$ ) sin llegar a establecer cuál algoritmo es el mejor, sin embargo, el algoritmo de la FMF logró detectar el $21.8 \%$ de los casos en comparación con el $21.5 \%$ que alcanzó a detectar el algoritmo de VP, una diferencia que, aunque pequeña, debe ser tenida en cuenta a la hora de escoger un algoritmo específico (36).

Teniendo en cuenta la evidencia presentada se refleja que, aunque la mayoría de los algoritmos utilizan los mismos parámetros de evaluación: antecedentes maternos, datos obtenidos del examen físico, la medición el IP-AtU y parámetros bioquímicos, los resultados en cuanto a la tasa de detección son similares (37); las diferencias entre estos, las cuales deben ser tenidas en cuenta por el médico tratante a la hora de seleccionar uno y aplicarlo en su práctica clínica, están dadas por variables como: el tamaño de la población en la que ha sido estudiada, la etnia y los resultados de acuerdo con la sensibilidad para el desarrollo de PE temprana, validación y accesibilidad reportados. De todos los algoritmos mencionados, el único que se encontró de libre acceso en internet para su aplicación fue el acoplado por la FMF (también es el que más estudios de validación tiene).

Por último, la implementación rutinaria de estos algoritmos implica un mayor costo, pues el uso de pruebas bioquímicas y la medición del IP-AtU requieren de mayor número de personas, tiempo y dinero. Hay escenarios sin disponibilidad de máquinas para medir la PAPP-A o el Factor de Crecimiento Placentario (PlGF), lo cual impide la aplicación total para la detección propuesta por estos algoritmos. Ante esta situación, Wright et al. (38) diseñaron un modelo 
de dos pasos, en el cual se hace tamizaje en un primer momento teniendo en cuenta solo factores de riesgo maternos o estos factores y la medición de la PAM y el IP-AtU, o tomando los factores y la medición de la PAM y el PlGF; y en un segundo momento aplicando una combinación de factores maternos y la medición de PAM, IP-AtU y PlGF solo para un subgrupo de población seleccionada como de alto riesgo. Se evidencia que, en el tamizaje en el primer momento entre los factores maternos, la PAM y el IP-AtU, la tasa de detección era de $91.2 \%$ para $\mathrm{PE}<32$ semanas y de $80.5 \%$ para $\mathrm{PE}<37$ semanas, y cuando se hacía el tamizaje con el triple test la tasa de detección era de $92.8 \%$ para $\mathrm{PE}<32$ semanas y de $81.3 \%$ para $\mathrm{PE}<37$ semanas. La TD es muy similar para ambas situaciones, y es posible reservar las mediciones de los parámetros bioquímicos. En Colombia, la medición del IP-AtU es factible y se encuentra validada por un estudio de cohorte en la ciudad de Bogotá en el que participaron 444 pacientes donde se encontró que el IP-AtU en el primer trimestre fue más alto en aquellas mujeres que desarrollaron preeclampsia en comparación con las que no, y puede ser una herramienta útil para realizar vigilancia estrecha en estas mujeres durante el control prenatal (17). Se puede hacer al momento de la ecografía programada para buscar aneuploidías y se hace con la misma máquina que realiza este procedimiento, por lo cual solo requeriría más disposición de tiempo y de práctica del personal para poder realizar el abordaje de esta manera $(39,40)$.

\section{Conclusión}

El Doppler de arterias uterinas se ha consolidado como una de las mejores herramientas para la predicción de preeclampsia, ya que es potencialmente útil, económico y no invasivo, por lo cual se debería evaluar su inclusión dentro de los diversos algoritmos que calculan el riesgo de PE entre las semanas 11 a 13+6. La validez de dicha prueba diagnóstica se ve reflejada en una sensibilidad del $47.8 \%$ y una especificidad del $92.1 \%$ para la detección de PE temprana; y una sensibilidad del $26.4 \%$ y especificidad del $93.4 \%$ para predecir el desarrollo de $\mathrm{PE}$ en cualquier etapa de la gestación (TFP de 6.6\%). La evidencia demuestra tasas TD $\geq 90 \%$ alcanzadas por distintos algoritmos que miden en conjunto el IP-AtU con otras variables. Colombia presenta una prevalencia de preeclampsia superior a la de países desarrollados, lo cual hace necesario un tamizaje efectivo que permita un diagnóstico oportuno, en el mejor de los casos, preventivo. El médico general, quien es el principal encargado de realizar el control prenatal a la población general, debe conocer la utilidad de esta herramienta para poder ofrecer un abordaje temprano y oportuno, solo así se disminuirán los posibles desenlaces desfavorables secundarios en estas pacientes.

\section{Conflictos de intereses}

Los autores declaran no tener conflictos de intereses.

\section{Referencias}

1. Lakshmy S, Ziyaulla T, Rose N. The need for implementation of first trimester screening for preeclampsia and fetal growth restriction in low resource settings. J Matern-Fetal Neo M [Internet]. 2020;34(24):4082-9. doi: https://doi.org/10.1080/147 67058.2019.1704246

2. Instituto Nacional de Salud; Boletín Epidemiológico Semanal [Internet]. Semana Epidemiológica 23.. 2019. Recuperado a partir de: https://www.ins.gov.co/ buscador-eventos/BoletinEpidemiologico/2019\%20 Bolet $\% \mathrm{C} 3 \% \mathrm{ADn} \% 20$ epidemiol $\% \mathrm{C} 3 \% \mathrm{~B} 3$ gico $\% 20$ semana\%2023.pdf

3. El-Sayed AAF. Preeclampsia: A review of the pathogenesis and possible management strategies based on its pathophysiological derangements. Taiwan J Obstet Gynecol [Internet]. 2017;56(5):5938. doi: https://doi.org/10.1016/j.tjog.2017.08.004

4. Sotiriadis A, Hernandez-Andrade E, da Silva Costa F, Ghi T, Glanc P, Khalil A, et al. ISUOG Practice Guidelines: Role of ultrasound in screening for and follow-up of pre-eclampsia. Ultrasound Obstet Gynecol. [Internet]. 2019;53(1):7-22. doi: https://doi. org/10.1002/uog.20105

5. Campbell S, Griffin DR, Pearce JM, Diaz-Recasens J, Cohen-Overbeek TE, Willson K, et al. New doppler technique for assessing uteroplacental blood flow. Lancet [Internet]. 1983;321(8326):675-677. doi: https://doi.org/10.1016/s0140-6736(83)91970-0

6. Acolet D, Springett A, Golightly S. Perinatal Mortality 2006 [Internet]. Confidential Enquiry into Maternal and Child Health; 2008. Recuperado a partir de: https://www.oaa-anaes.ac.uk/assets/ managed/editor/ File/Reports/2006_Perinatal_mortality.pdf

7. Farré MT, Borrell A, Ravenau W, Azulay M, Cararach V, Fortuny A. Estudio Doppler de las arterias uterinas: predicción de complicaciones perinatales. Prog Obstet Ginecol [Internet]. 2001;44(12):537-43. doi: https:// doi.org/10.1016/S0304-5013(01)75714-7

8. Papageorghiou A, Yu CKH, Bindra R, Pandis G, Nicolaides KH. Multicenter screening for preeclampsia and fetal growth restriction by transvaginal 
uterine artery Doppler at 23 weeks of gestation. Ultrasound Obstet Gynecol. 2001;18(5):441-9. doi: https://doi.org/10.1046/j.0960-7692.2001.00572.x

9. Martin AM, Bindra R, Curcio P, Cicero S, Nicolaides KH. Screening for pre-eclampsia and fetal growth restriction by uterine artery Doppler at 11-14 weeks of gestation. Ultrasound Obstet Gynecol [Internet]. 2001;18(6):583-6. doi: https://doi.org/10.1046/ j.0960-7692.2001.00594.x

10. Velauthar L, Plana MN, Kalidindi M, Zamora J, Thilaganathan B, Illanes SE, et al. First-trimester uterine artery Doppler and adverse pregnancy outcome: A meta-analysis involving 55974 women. Ultrasound Obstet Gynecol [Internet]. 2014;43(5): 500-7. doi: https://doi.org/10.1002/uog.13275

11. von Dadelszen P, Payne B, Li J, Ansermino JM, Pipkin F, Côté AM, et al. Prediction of adverse maternal outcomes in pre-eclampsia: development and validation of the fullPIERS model. Lancet [Internet]. 2011;377(9761):219-27. doi: https://doi.org/10.1016/ S0140-6736(10)61351-7

12. Chaiworapongsa T, Chaemsaithong P, Yeo L, Romero R. Pre-eclampsia part 1: current understanding of its pathophysiology. Nat Rev Nephrol [Internet]. 2014;10:466-80. doi: https://doi.org/10.1038/ nrneph.2014.102

13. Orabona R, Donzelli C, Falchetti M, Santoro A, Valcamonico A, Frusca T. Placental histological patterns and uterine artery Doppler velocimetry in pregnancies complicated by early or late preeclampsia. Ultrasound Obstet Gynecol [Internet]. 2016;47(5):580-5. doi: https://doi.org/10.1002/ uog.15799

14. Bujold E, Roberge S, Lacasse Y, Bureau M, Audibert F, Marcoux S, et al. Prevention of Preeclampsia and Intrauterine Growth Restriction with Aspirin Started in Early Pregnancy: A Meta-Analysis. Obstet Gynecol [Internet]. 2010;116(2p1):402-14. doi: https://doi. org/10.1097/aog.0b013e3181e9322a

15. Dugoff L, Lynch AM, Cioffi-Ragan D, Hobbins JC, Schultz LK, Malone FD, et al. First trimester uterine artery Doppler abnormalities predict subsequent intrauterine growth restriction. Am J Obstet [Internet]. 2005;193(3):1208-12. doi: https://doi.org/10.1016/j. ajog.2005.06.05

16. Plasencia W, Barber M, Álvarez E, Segura J, Valle L, Garcia-Hernandez J. Comparative Study of Transabdominal and Transvaginal Uterine Artery Doppler Pulsatility Indices at 11-13 + 6 Weeks. Hypertens Pregnancy. 2011;30(4):414-20. doi: https:// doi.org/10.3109/10641955.2010.506232

17. Cortés-Yepes H. Doppler de arterias uterinas en el primer trimestre del embarazo para la detección de los trastornos hipertensivos asociados con el embarazo: estudio de cohorte. Bogotá (Colombia) 2007-2008. Rev Colomb Obstet Ginecol. [Internet]. 2009;60(4):328-33. doi: https://doi.org/10.18597/ rcog. 315

18. Khalil A, Nicolaides KH. How to record uterine artery Doppler in the first trimester. Ultrasound Obstet Gynecol [Internet]. 2013;42:478-9. doi: https://doi. org/10.1002/uog.12366

19. Witlin AG, Saade GR, Mattar F, Sibai BM. Predictors of neonatal outcome in women with severe preeclampsia or eclampsia between 24 and 33 weeks' gestation. Am J Obstet [Internet]. 2000;182(3):60711. doi: https://doi.org/10.1067/mob.2000.104224

20. Demers ME, Dubé S, Bourdages M, Gasse C, Boutin A, Girard $\mathrm{M}$ et al. Comparative Study of Abdominal Versus Transvaginal Ultrasound for Uterine Artery Doppler Velocimetry at 11 to 13 Weeks. J Ultrasound Med [Internet]. 2018;37(7):1771-6. doi: https://doi. org/10.1002/jum.14530

21. Tayyar A, Guerra L, Wright A, Wright D, Nicolaides $\mathrm{KH}$. Uterine artery pulsatility index in the three trimesters of pregnancy: Effects of maternal characteristics and medical history. Ultrasound Obstet Gynecol [Internet]. 2015;45(6):689-97. doi: https:// doi.org/10.1002/uog.14789

22. Rivas M, González X, Guevara H. Valores de referencia del índice de pulsatilidad de la arteria uterina durante el embarazo. Rev Obstet Ginecol Venez [Internet]. 2016;76(3):225-31. Recuperado a partir de: http://ve.scielo.org/scielo.php?script=sci arttext\&pid=S0048-77322016000400002\&lng=es

23. Ferreira AEGMT, Mauad Filho F, Abreu PSG, Mauad FM, Araujo Júnior E, Martins WP. Reproducibility of first- and second-trimester uterine artery pulsatility index measured by transvaginal and transabdominal ultrasound. Ultrasound Obstet Gynecol [Internet]. 2015;46(5):546-52. doi: https://doi.org/10.1002/ uog. 14762

24. Peixoto A, Rodrigues Da Cunha Caldas T, Tonni G, Almeida Morelli P, Santos L, Martins W, et al. Reference range for uterine artery Doppler pulsatility index using transvaginal ultrasound at 20-24w6d of gestation in a low-risk Brazilian population. J Turk Ger Gynecol Assoc. 2016;17:16-20. doi: https://doi. org/10.5152/jtgga.2016.16192

25. Gómez O, Martínez JM, Figueras F, Del Río M, Borobio V, Puerto B, et al. Uterine artery Doppler at 11-14 weeks of gestation to screen for hypertensive disorders and associated complications in an unselected population. Ultrasound Obstet Gynecol [Internet]. 2005;26(5):490-4. doi: https://doi. org/10.1002/uog.1976

26. Poon LC, Shennan A, Hyett JA, Kapur A, Hadar E, Divakar H, et al. The International Federation 
of Gynecology and Obstetrics (FIGO) initiative on pre-eclampsia: A pragmatic guide for first-trimester screening and prevention. Int $\mathrm{J}$ Gynecol Obstet [Internet]. 2019;145(S1):1-33. doi: https://doi. org/10.1002/ijgo.12802

27. O'Gorman N, Wright D, Syngelaki A, Akolekar R, Wright A, Poon LC, et al. Competing risks model in screening for preeclampsia by maternal factors and biomarkers at 11-13 weeks gestation. Am J Obstet Gynecol [Internet]. 2016;214(1):103.e1-12. doi: https://doi.org/10.1016/j.ajog.2015.08.034

28. O'Gorman N, Wright D, Poon LC, Rolnik DL, Syngelaki A, Wright A, et al. Accuracy of competingrisks model in screening for pre-eclampsia by maternal factors and biomarkers at 11-13 weeks' gestation. Ultrasound Obstet Gynecol [Internet]. 2017;49(6):751-755. doi: https://doi.org/10.1002/ uog. 17399

29. O'Gorman N, Wright D, Poon L, Rolnik D, Syngelaki A, de Alvarado $\mathrm{M}$, et al. Multicenter screening for pre-eclampsia by maternal factors and biomarkers at 11-13 weeks' gestation: Comparison with NICE guidelines and ACOG recommendations. Ultrasound Obstet Gynecol [Internet]. 2017;49(6):756-60. doi: https://doi.org/10.1002/uog.17455

30. ACOG. First-Trimester Risk Assessment for EarlyOnset Preeclampsia. Committee opinion No. 638. Obstet Gynecol. 2015;126:e25-7. doi: https://doi. org/10.1097/aog.0000000000001049

31. National Collaborating Centre for Women's and Children's Health (UK). Hypertension in Pregnancy: The Management of Hypertensive Disorders During Pregnancy. London: RCOG Press; 2010 Aug. (NICE Clinical Guidelines, No. 107.) Recuperado a partir de: https://www.ncbi.nlm.nih.gov/books/NBK62652/

32. Akolekar R, Syngelaki A, Poon L, Wright D, Nicolaides K. Competing Risks Model in Early Screening for Preeclampsia by Biophysical and Biochemical Markers. Fetal Diagn Ther. 2013;33(1):815. doi: https://doi.org/10.1159/000341264

33. Rolnik DL, Wright D, Poon LCY, Syngelaki A, O'Gorman N, de Paco Matallana C, et al. ASPRE trial: Performance of screening for preterm preeclampsia. Ultrasound Obstet Gynecol [Internet]. 2017;50(4):492-5. doi: https://doi.org/10.1002/ uog. 18816

34. Skråstad R, Hov G, Blaas $H$, Romundstad $P$, Salvesen K. Risk assessment for preeclampsia in nulliparous women at 11-13 weeks gestational age: Prospective evaluation of two algorithms. BJOG [Internet]. 2015;122(13):1781-8. doi: https://doi. org/10.1111/1471-0528.13194

35. Lakovschek IC, Ulrich D, Jauk S, Csapo B, Kolovetsiou-Kreiner V, Mayer-Pickel K, et al. Risk assessment for preterm preeclampsia in first trimester: Comparison of three calculation algorithms. Eur J Obstet Gynecol [Internet]. 2018;231:241-7. doi: https://doi.org/10.1016/j.ejogrb.2018.11.006

36. Duley L. The Global Impact of Pre-eclampsia and Eclampsia. Semin Perinatol [Internet]. 2009;33(3):130-7. doi: https://doi.org/10.1053/j. semperi.2009.02.010

37. O'Gorman N, Nicolaides K, Poon L. The Use of Ultrasound and other Markers for Early Detection of Preeclampsia. Womens Health. 2016;12(2):199-207. doi: https://doi.org/10.2217/whe.15.95

38. Wright A, Wright D, Syngelaki A, Georgantis A, Nicolaides KH. Two-stage screening for preterm preeclampsia at 11-13 weeks' gestation. Am J Obstet Gynecol [Internet]. 2019;220(2):197.e1-11. doi: https://doi.org/10.1016/j.ajog.2018.10.092

39. Tan MY, Syngelaki A, Poon LC, Rolnik DL, O'Gorman N, Delgado JL, et al. Screening for preeclampsia by maternal factors and biomarkers at 11-13 weeks' gestation. Ultrasound Obstet Gynecol [Internet]. 2018;52(2):186-195. doi: https://doi. org/10.1002/uog.19112

40. Ridding G, Schluter PJ, Hyett JA, McLennan AC. Uterine Artery Pulsatility Index Assessment at 11-13 Weeks' Gestation. Fetal Diagn Ther [Internet]. 2014;36:299-304. doi: https://doi. org/10.1159/000361021 